\title{
Behaviour of materials under shock loading conditions
}

\author{
S K SIKKA \\ High Pressure Physics Division, Bhabha Atomic Research Centre, Bombay 400085, India \\ Abstract. Shock wave research is a multidisciplinary field. In materials science, it is used \\ to study equation-of-state, phase transitions and mechanical properties. In material processing, \\ synthesis, powder compaction, shock sintering, shock welding etc. have been the prominent \\ applications. We have been doing shock wave research at Trombay during the last two \\ decades. Recently, we have built a single-stage gas gun to generate shock pressures in samples. \\ In this paper, we describe this facility and some work done on the interpretation of \\ shock-induced phase transitions.
}

Keywords. Shock loading; phase transitions; behaviour of materials; single-stage gas gun.

\section{Introduction}

Research on shock wave effects in materials is of importance in both basic and applied sciences. In basic sciences, this provides a test to the theoretical models of cohesion of matter. In applied sciences, vital inputs for computer simulations of a variety of situations (reactor accidents, effects of nuclear explosions, hypervelocity impact etc.) are generated by shock wave experiments. It is also possible to produce and process novel materials with useful properties under shock conditions.

The current laboratory and field techniques of producing shocks can subject a material to pressures up to $500 \mathrm{TPa}(1 \mathrm{TPa} \equiv 10 \mathrm{Mbar} ; 1 \mathrm{Mbar} \equiv 100 \mathrm{GPa} ; 1 \mathrm{GPa} \equiv$ $10^{10}$ dynes $/ \mathrm{cm}^{2}$ ). The material behaviour, in this pressure regime (from low to high pressures) ranges from elastic, plastic, melting and other phase transitions, electron structure changes, pressure and temperature ionization of inner electronic shells and finally to ideal gas behaviour. At BARC, for the last two decades, we have been investigating some of these phenomena by first principles electron theory methods. The earlier work was reviewed in Godwal et al (1983) and Sikka $(1985,1986)$. We had also carried out numerical simulation of the effects of shock wave propagation in materials (Gupta 1980) and applied this to understand the effects of Pokaran underground nuclear explosion on surrounding rocks (Chidambaram et al 1985). More recently, we have concentrated on the interpretation of shock-induced phase changes and have built up experimental facilities to generate shock waves in samples. In this paper, we dwell on these recent developments. A brief description of the basics of the shock wave physics is first presented.

\section{Shock waves}

A shock wave is a large amplitude disturbance, moving with supersonic speed, across which the stress, density and other physical properties of the material change in a near discontinuous manner (McQueen et al 1970). A shock wave can be generated by various methods including explosive loading, plate impact and energy deposition. A typical plate impact is illustrated in figure 1 . When a high velocity projectile strikes 


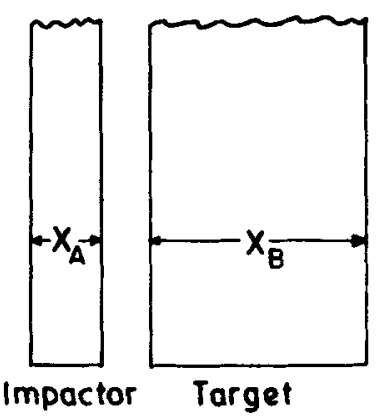

(a)

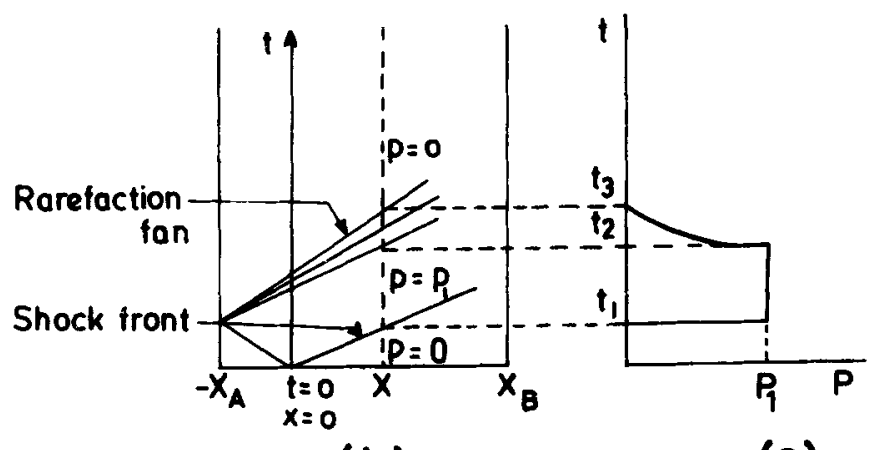

(b)

(c)

Figure 1. (a) Impact of flyer with a target. (b) $x-t$ diagram for the impact and (c) stress profile at the lagrangian point $x$.

a target, a shock wave is generated in the target material. This traverses through the target in about a microsecond. In the ideal case of steady shock front where it connects an undistributed state to a uniform shocked state, assuming that these two are equilibrium states, it can be proved, using the laws of conservation of mass, momentum and energy, that across the shock front the following relations hold:

$$
\begin{aligned}
\rho_{0} U_{s} & =\rho_{1}\left(U_{s}-U_{p}\right), \\
\sigma_{1}-\sigma_{0} & =\rho_{0} U_{s} U_{p}, \\
E_{1}-E_{0} & =1 / 2\left(\sigma_{1}+\sigma_{0}\right)\left(V_{0}-V_{1}\right) .
\end{aligned}
$$

Here $U_{s}$ is the shock front velocity, $U_{p}$ the particle velocity in the compressed region, $\sigma, E, \rho$ and $V$ are the longitudinal stress, specific internal energy, density and specific volume respectively. Suffixes 1 and 0 represent the quantities in the shocked and unshocked regions.

In a series of shock wave experiments, each compressing material from a fixed $V_{0}$ and $E_{0}$, with different magnitudes of stress, a different change of volume will be obtained. A locus of these different $\left(\sigma_{1}, V_{1}\right)$ states is known the Hugoniot of the material, centred at $\left(\sigma_{0}, V_{0}\right)$. In an impact-induced shock wave experiment, one usually measures the projectile velocity and the shock velocity in the target. The induced particle velocity in the target can be inferred by the impedance mismatch condition 

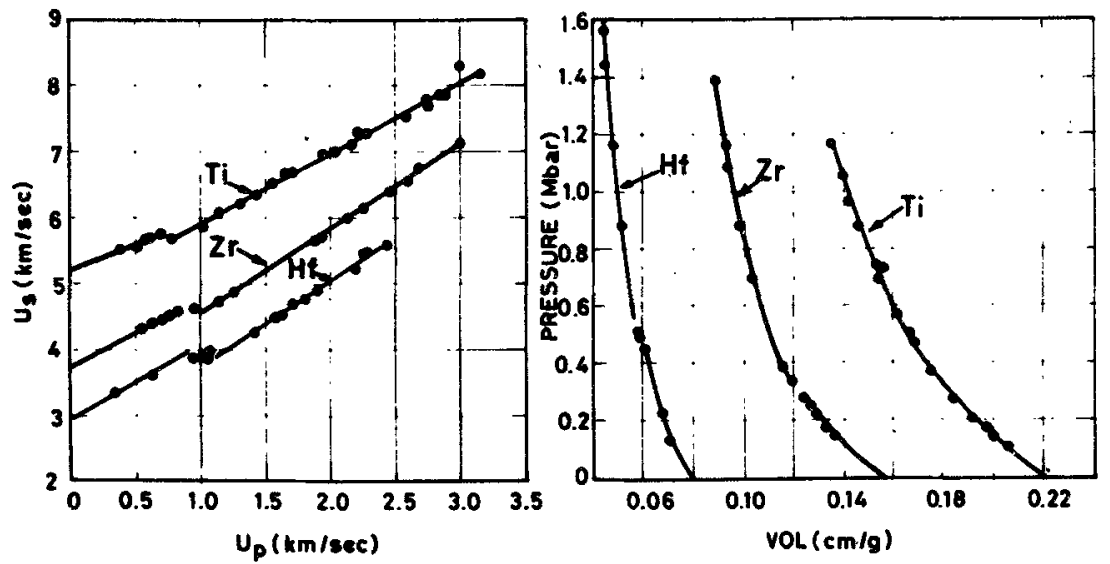

Figure 2. Shock wave data on $\mathrm{Ti}, \mathrm{Zr}$ and $\mathrm{Hf}$. (a) $U_{s}-U_{p}$ plots and (b) $P$ vs $V$ curves (from MacQueen et al 1970).

(it is exactly half when the impactor and target are made of the same material). The stress can then be deduced from equation (2) and hence a Hugoniot point derived. A Hugoniot can either be plotted in the $\sigma-V$ plane or the more popular, $U_{s}-U_{p}$ plane. This latter representation is quite attractive, as shock velocity, being in essence a differential quantity, (rearranging equations (1) and (2)),

$$
U_{s}=\left|\frac{V_{0}\left(\sigma_{1}-\sigma_{0}\right)}{V_{0}-V_{1}}\right|^{1 / 2}
$$

is a very sensitive parameter. Thus, changes in the equation-of-state due to various physical phenomena, barely discernible in $\sigma-V$ curves are easily seen in $U_{s}-U_{p}$ plots (e.g. see figure 2).

\section{Some differences from static pressure experiments}

One can express the longitudinal shock stress, assuming that the plane shock is propagating in the $x$ direction $\left(\sigma_{1}-\sigma_{0} \equiv \sigma_{x}\right)$, as

$$
\begin{aligned}
\sigma_{x} & =\frac{1}{3}\left(\sigma_{x}+2 \sigma_{z}\right)+\frac{1}{3}\left[2\left(\sigma_{x}-\sigma_{z}\right)\right] \\
& =P+(4 / 3) \tau .
\end{aligned}
$$

Here $\sigma_{z}\left(=\sigma_{y}\right)$ is the lateral stress, $\tau=\frac{1}{2}\left(\sigma_{x}-\sigma_{z}\right)$, the maximum resolved shear stress and $P$ the hydrostatic stress component. Thus, the Hugoniot differs from the hydrostat and shear is always present in dynamic pressure experiments compared to static pressure ones. This is of importance for phase transitions, as most of the high pressure transformations are of martensitic or displacive type and shear will add in inducing these transitions (e.g. in the case of $\alpha-\omega$ transition in $\mathrm{Ti}$ and $\mathrm{Zr}$, the shear component brings down the transition pressure from 6-7 to 2 GPa (Zilbershtein et al 1975)).

The shock Hugoniot and hydrostat are also different from the isotherm and isentrope passing through the same initial state as shown in figure 3. The amounts of thermal energy on shock loading and unloading (i.e. irreversible heating) are also shown. Further, the amount of compression produced in single shock experiments is 


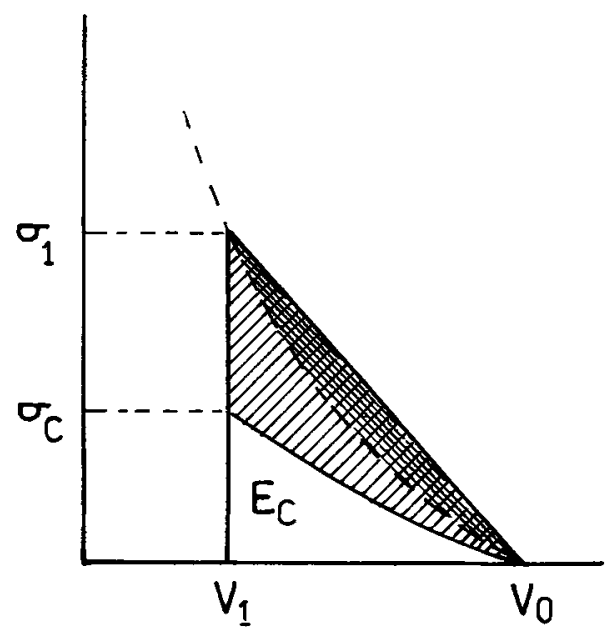

Figure 3. Interpretation of equation (3). $\sigma_{c}$ and $E_{c}$ are zero degree contributions. Double hatched area denotes irreversible heating and Hugoniot heating is the area $\sigma_{1} \sigma_{c} v_{0}$.

Table 1. Various pressure contributions to Hugoniot of Al (from Godwal et al 1977).

\begin{tabular}{lcrccc}
\hline$V / V_{0}$ & $\begin{array}{c}T_{H} \\
(\mathrm{eV})\end{array}$ & $\begin{array}{c}P_{\mathrm{c}} \\
(\mathrm{GPa})\end{array}$ & $\begin{array}{c}P_{1 T} \\
(\mathrm{GPa})\end{array}$ & $\begin{array}{c}P_{e} \\
(\mathrm{GPa})\end{array}$ & $\begin{array}{c}P_{\mathrm{c}}+P_{1 T}+P_{e} \\
(\mathrm{GPa})\end{array}$ \\
\hline 0.9 & 0.043 & 8.3 & 1.06 & - & 9.4 \\
0.85 & 0.084 & 14.4 & 3.5 & - & 17.9 \\
0.75 & 0.09 & 33.5 & 4.1 & - & 37.6 \\
0.65 & 0.284 & 69.2 & 18.2 & 2.2 & 89.6 \\
0.60 & 0.554 & 98.4 & 39.5 & 4.7 & 142.6 \\
0.55 & 1.10 & 141.0 & 84.6 & 13.8 & 239.4 \\
\hline
\end{tabular}

$T_{H}$, Hugoniot temperature; $P_{c}$, zero degree pressure; $P_{1 T}$, ionic thermal pressure; $\boldsymbol{P}_{\infty}$ electronic excitation pressure

limited as at higher shock strengths, more energy goes into heating. Table 1 shows the magnitude of these effects. Also, on the $P, V, T$ surface, the Hugoniot chalks out a path different from an isotherm in static pressure experiments (e.g. in figure 4, note that (i) the transition pressure can be different for the same phase change and (ii) different phases may be encountered along an isotherm and the Hugoniot).

The duration of the shock waves in samples is small $(\approx$ microsecond) and further the rise time of the shock front is typically a few nanoseconds. Both these factors can influence the kinetics of phase changes. The time available may not be sufficient for the new phase to grow so that it may go undetected and thus the sample size may be of importance in some cases. As the shock loading rate is $10^{8}$ times higher than in static experiments, in extreme cases, again the phase transition may be altogether missed in shock experiments (see figure 5 for a time-pressure transformation diagram).

However, in many cases, it has been observed that polymorphic phase transitions, which frequently exhibit slow kinetics under static loading, occur very rapidly in shock experiments. A classic example is the $\alpha-\varepsilon$ transition in iron, which was first detected in dynamic experiments (Minshall 1955). This aspect of rapid growth of 


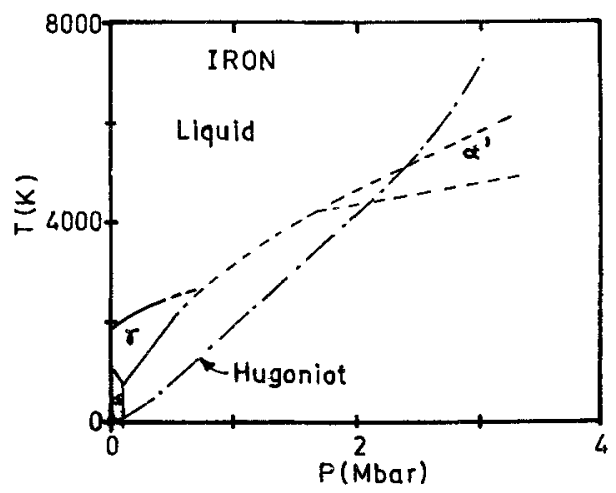

Figure 4. Pressure temperature phase diagram of iron along with the Hugoniot curve (adopted from Ross 1990 and Brown and MacQueen 1986).

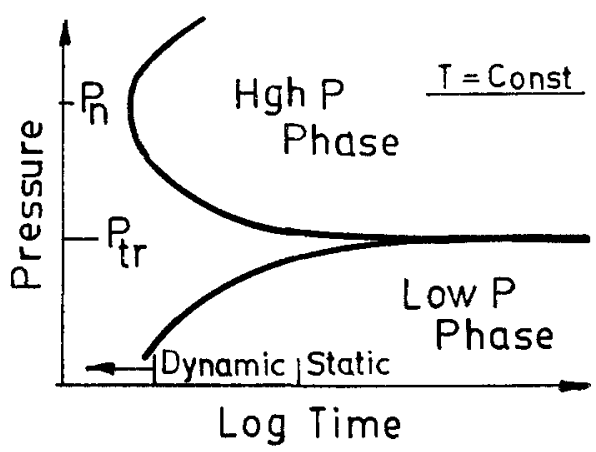

Figure 5. Pressure-time transformation diagram (from Jeanloz 1987). If a time-pressure path is to the left of the nose pressure $\left(P_{n}\right)$, then transformation does not occur.

phases under shocks is not fully understood. However, the most common explanation offered is that the shock passage is accompanied by creation of an enormous number of defects in the samples. These serve as nucleation sites for the growth of the new phase.

\section{A single-stage gas gun}

The functional block diagram of the gun facility (Gupta et al 1990) at Trombay is given in figure 6. Its main parts are a breech, a barrel and a target catcher system. The breech is capable of holding a high pressure gas (nitrogen or helium) and has a breech opening mechanism for allowing the gas to flow behind the projectile within a few milliseconds. Two kinds of breeches (figure 7) are used: a wrap-around breech designed for below 200 bars pressure and a double diaphragm breech up to a pressure of 410 bars. In the first one, firing is accomplished by injecting a small amount of gas behind the projectile causing it to move past the ports, initially blocked by the projectile. In the double diaphragm breech, two diaphragms are used which can withstand slightly more than half the reservoir pressure. Firing is done by exhausting the region between the diaphragms (initially pressurized to half the pressure) so that each diaphragm in turn experiences the full gas pressure. 


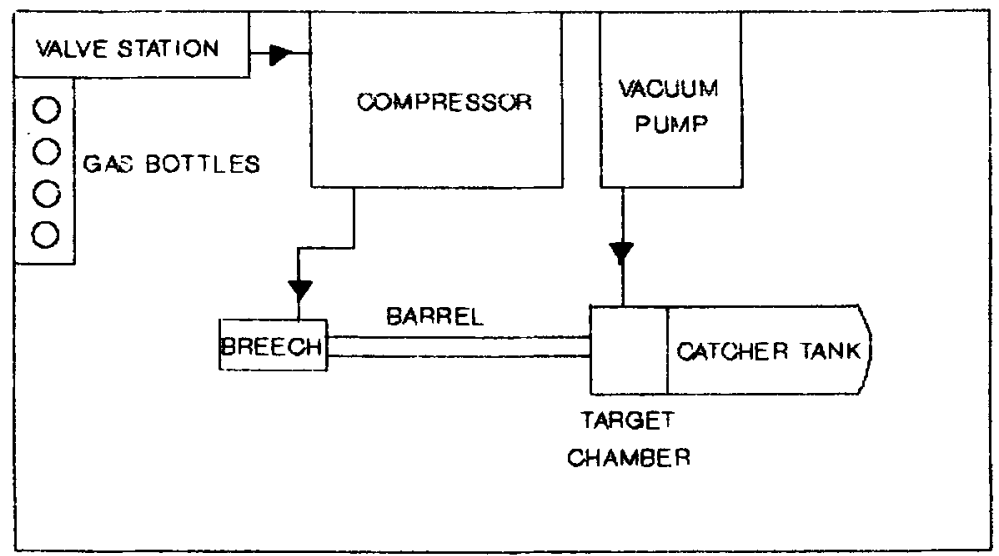

Figure 6. Schematic of thẻ gas gun facility at BARC.

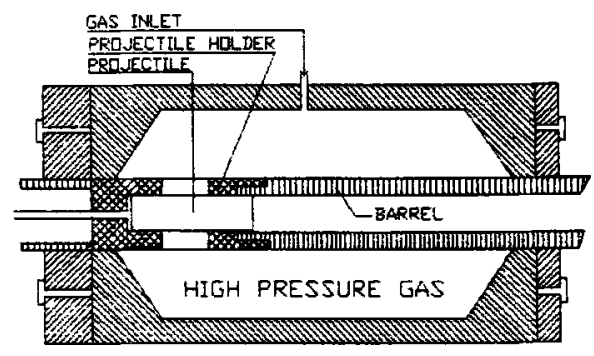

WRAP ARDUND BREECH

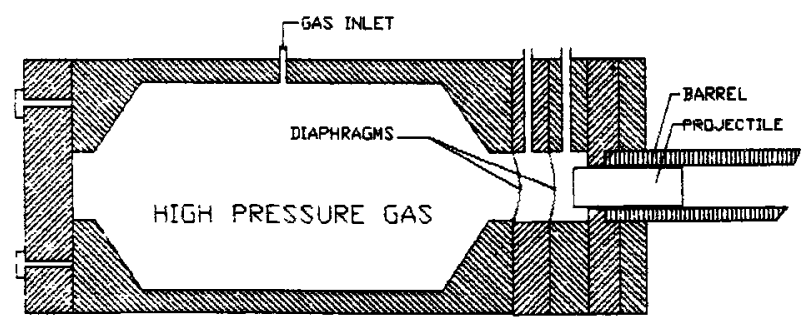

DDUBLE DIAPHRAGM BREECH

Figure 7. (a) Wrap-around breech and (b) double diaphragm breech.

The barrel is $3 \mathrm{~m}$ long with an inside diameter of $63.5 \mathrm{~mm}$. The ID of the barrel is uniform within 20 microns. Both planar and inclined impacts (for compression shear measurements) can be obtained. For the latter, the barrel has been slotted and a key is provided on the projectile.

The catcher system consists of two sections. One section (the target chamber) is permanently mounted to the muzzle end of the barrel. The second section is a projectile stopping system. A thick mylar diaphragm separates the two. The supporting systems include vacuum pumps for evacuating the barrel and target chamber and a compressor for providing the required gas pressure. A remote control unit allows firing of the projectile in manual or auto mode. A photograph of the facility is shown in figure 8 . 


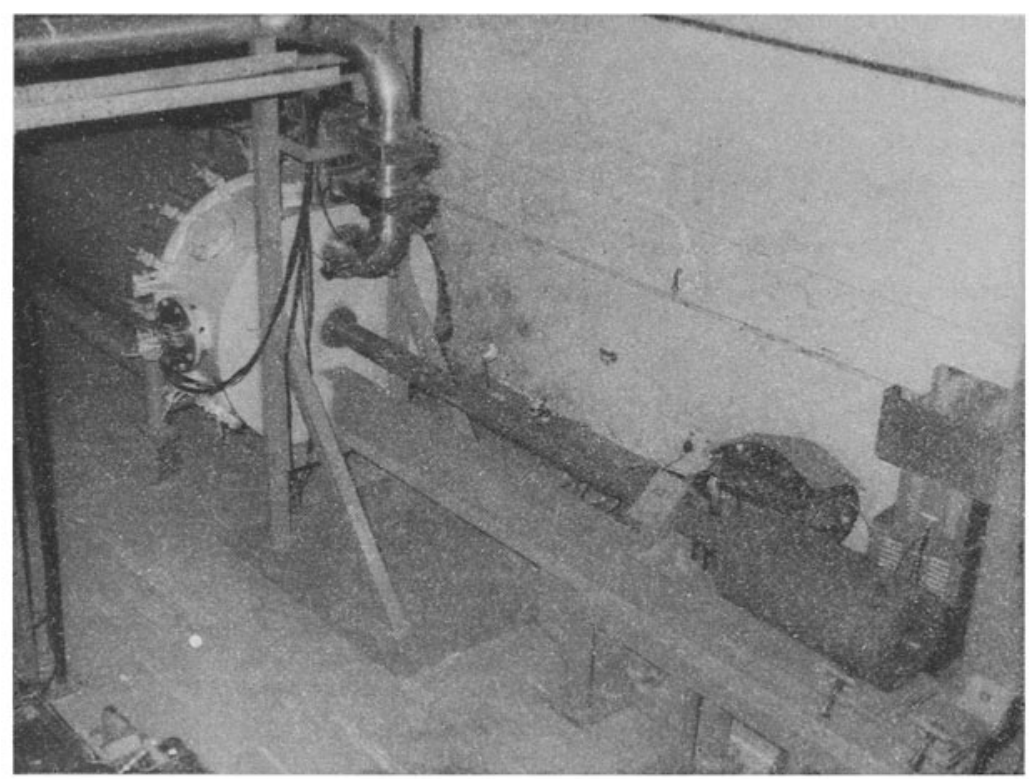

Figure 8. Photograph of the gas gun facility at Trombay (Gupta et al 1990).

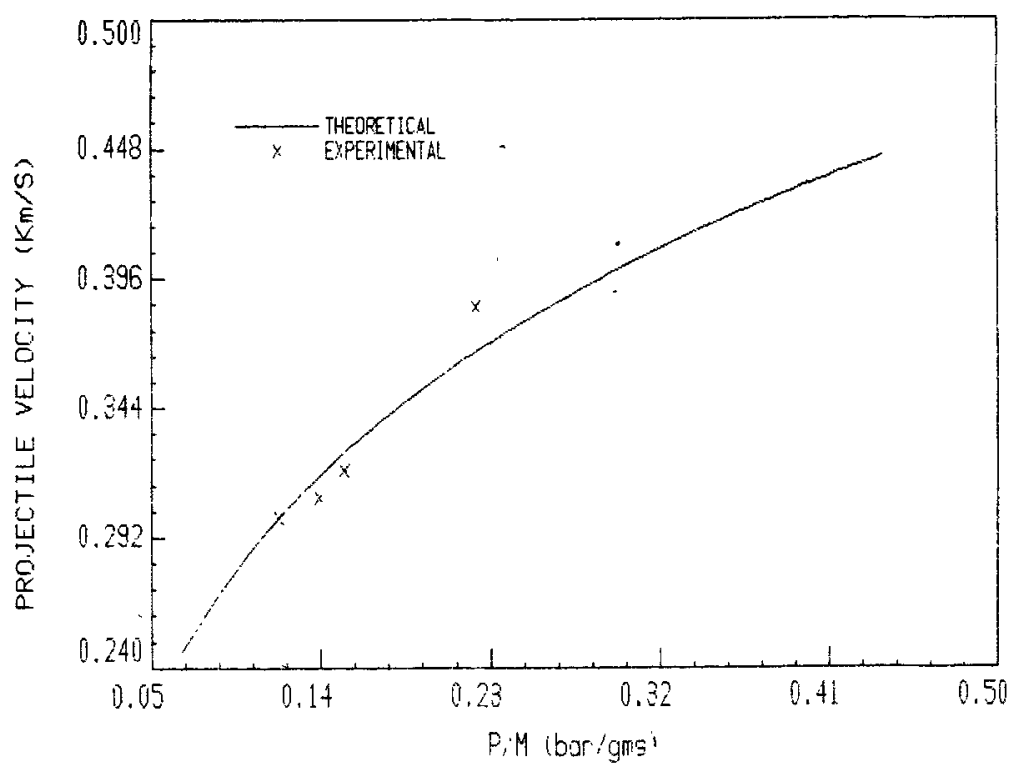

Figure 9. Projectile velocity versus breech pressure/projectile mass $(P / M)$ curve.

By selecting the initial gas pressure in the breech, we can get projectile (mass $\approx 300 \mathrm{~g}$ ) velocities up to $1 \mathrm{~km} / \mathrm{s}$. When this high velocity projectile impacts a target, a shock pressure (up to $40 \mathrm{GPa}$, depending upon the impedances of the impactor and the target materials) is generated. This shock moves through the target in about a microsecond and all the measurements are completed during this short period of time, using fast storage electronics. 
We have developed instrumentation to measure the shock velocity, the particle velocity and shock pressure profile as a function of time. Diagnostics have also been built for measuring the projectile velocity and the tilt of the impactor. Figure 9 displays a comparison of the expected and measured projectile velocities. It shows that the gun is performing as expected.

\section{Interpretation of shock-induced transitions in solids}

Shock-induced phase transitions in solids have been an active area of research ever since the discovery of the $\alpha-\varepsilon$ transition in iron. These are usually observed by (i) measurement of shock compression curve (indicated by breaks or discontinuities in $U_{s}-U_{p}$ curves), (ii) shock synthesis experiments using a recovery system, when these phase changes are irreversible on shock release, (iii) wave profile measurements of pressure or particle velocity (indicated by a two-wave structure) and (iv) sound velocity measurements in shock overtake experiments, which again display abrupt changes on phase transitions. All the techniques are macroscopic in nature and the exact nature of a phase transformation has to be inferred by indirect means. It may be noted that the use of microscopic techniques like Raman and X-ray diffraction studies has been demonstrated under shock conditions (Johnson et al 1970; Wark et al 1987), but there are hardly any applications yet in the field of shock-induced phase transitions.

The indirect means to understand shock transitions are (i) direct comparison with static high pressure data and (ii) resort to theoretical calculations, where one is now able to evaluate accurately the small Gibbs-free energy changes between different phases. In the next section, we demonstrate these by some of the work done by us.

\section{The valence $2-3$ transition in shocked ytterbium}

Ytterbium $(Z=70)$ is divalent in solid state at ambient conditions, unlike its neighbouring rare earth elements, which are trivalent. Takemura and Syassen (1985) measured its $p-v$ curve using powder $\mathrm{X}$-ray diffraction up to $30 \mathrm{GPa}$, and found that it anomalously deviates from the calculated $p-v$ curve for the divalent configuration and attained even smaller volume than the reference trivalent $\mathrm{Lu}$. This was taken to indicate mixed valent behaviour, which corroborated their earlier absorption edge experiments (Syassen and Vortmann 1981). They found that the valence of $\mathrm{Yb}$ increases gradually from 2 at a normal pressure to 2.9 at $33 \mathrm{GPa}$. Shock wave measurements on Yb have been made by two groups (Gust and Royce 1973; Carter et al 1975). Both measurements show a discontinuity in the slope of $U_{s}-U_{p}$ curve and a stiffening of the Hugoniot at a compression of $V / V_{0}=0.58$ and a pressure of about $13-16 \mathrm{GPa}$. We examined, using band theory techniques, whether valence change of $\mathrm{Yb}$ observed in static experiments is also displayed under dynamic compression (Gyanchandani et al 1987).

We did this by theoretical construction of Hugoniots of trivalent and divalent states. The zero degree isotherms were first computed by the linear muffin tin orbital method (Skriver 1984) and the Birch equation. The Hugoniot curves were then derived by solving equation (3) in combination with a Debye-Gruneisen thermal contribution. The trivalent Hugoniot was properly recentred at the experimental $V_{0}$. Figure 10 compares these curves with experimental shock data. It shows very clearly that $\mathrm{Yb}$ displays a mixed valent behaviour between 4 and $16 \mathrm{GPa}$ and its valence $\approx 3$ beyond 


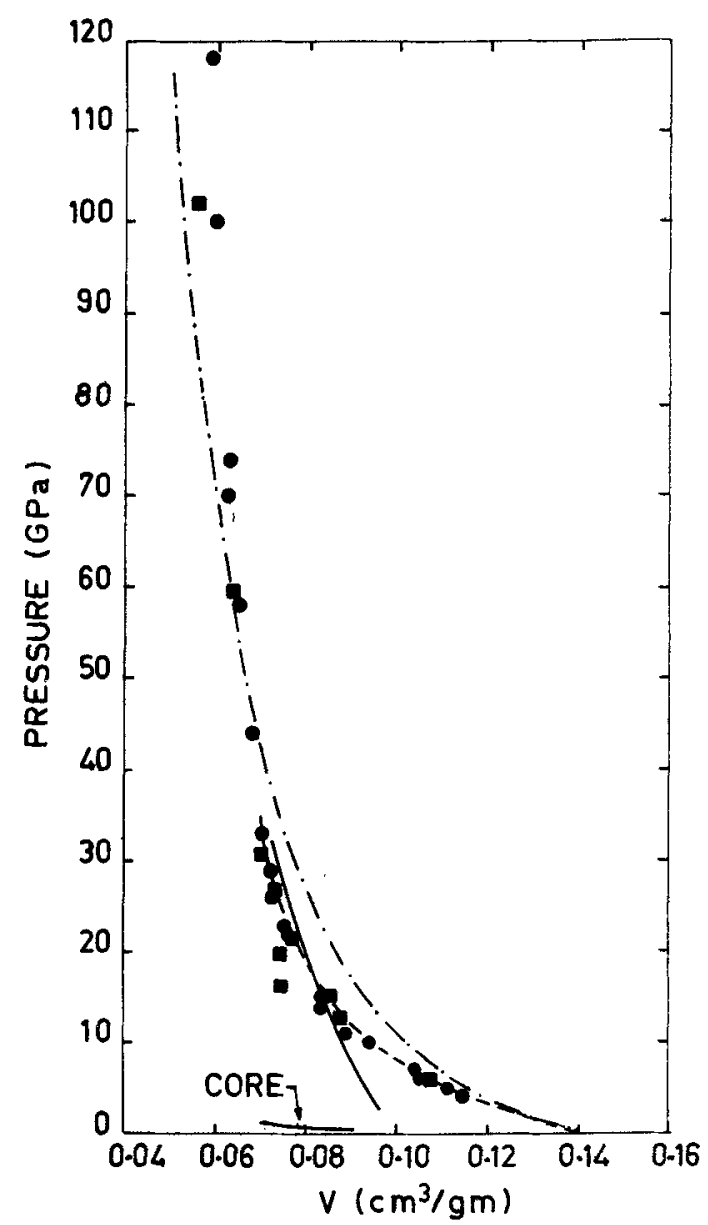

Figure 10. Observed and calculated Hugoniot of $\mathrm{Yb}$. $\square$ and $\mathbf{a r e}$ data from Gust and Royce (1973) and Carter et al (1975). — Calculated recentred trivalent Hugoniot. --.calcu.dted divalent Hugoniot.

this shock pressure. Also that this valence change is the principal cause of the stiffening of the shock Hugoniot after this pressure.

\section{On the shock discontinuities in $\mathrm{Ti}, \mathrm{Zr}$ and $\mathrm{Hf}$}

The $U_{s}-U_{p}$ plots, as measured by McQueen et al (1970), have already been shown in figure 2. There are discontinuities in these at pressures of 17, 26 and $40 \mathrm{GPa}$ for $\mathrm{Ti}, \mathrm{Zr}$ and $\mathrm{Hf}$ respectively. There have been many speculations regarding the nature of these. These authors assumed them to be transitions between the ambient condition! hcp $(\alpha)$ and the high temperature bcc $(\beta)$ phases in them, based on the observation of the bcc phase in the shock recovered samples of Ti. Subsequently, Pashkov and Polykova (1972) also reported the fixation of the bcc phase in shocked Zr. However, Kutsar and German (1976) found, instead, $\omega$ phase and explained the presence of the bcc phase as a result of a process occurring only at the surface of the specimens. Wave profile measurements by Kutsar (1982) and Kutsar et al (1984) place the transition pressures at 11.9 and $6.2-6.7 \mathrm{GPa}$ for $\mathrm{Ti}$ and $\mathrm{Zr}$ in much better agreement 
with static pressure values for the $\alpha-\omega$ phase change, which range from 2-7 GPa and 2.1-6.0 GPa for the two elements (see Sikka et al 1982 for more details). Kiselov and Falkov (1982) again, from the wave profile analysis, determined the transition pressure to be $6.0 \pm 0.3 \mathrm{GPa}$ for $\mathrm{Ti}$, and conjectured that the higher value (17 GPa) in Ti of McQueen et al was due to the use of thin samples so that steady-state shock conditions were not established.

In view of the inconsistency between the static pressure values for the $\alpha-\omega$ transition and the observed values for discontinuities in the data of McQueen et al, Carter (1973) had earlier suggested these to be an electronic transition. Guillermet (1987) recently analysed the Hugoniot data of $\mathrm{Zr}$ by thermodynamic methods and concludes that the transition at $26 \mathrm{GPa}$ in $\mathrm{Zr}$ is from hcp to bcc.

To clarify the above confused situation, we have performed first principles calculations of the equation-of-state and the analysis of the stability of the various phases in the pressure range of the experimental measurements. We have also looked into the possibility that the presence of the $\beta$-phase in the shock-recovered samples may be due to the shear banding which could cause heterogeneous heating in the samples, consequently transforming them to the high temperature phase (Gyanchandani et al 1989, 1990).

We have employed the density functional theory in the one-electron form and have approximated the total energy differences of crystal structures by the Andersen force theorem (Pettifor 1976; Mackintosh and Andersen 1980). The theorem states that the difference in the total energy at a given volume, between two structures (say I and II), is given by the differences in the sum of the one-electron eigenvalues, provided one uses for structure II, a frozen potential, obtained from a self-consistent calculation of structure I. A muffin-tin electrostatic correction for the non-sphericity of the WignerSeitz cell is also added. The one-electron eigenvalues were again determined using the linear muffin tin orbital method (Skriver 1984).

Computed energy differences at $0^{\circ} \mathrm{K}$ for $\mathrm{hcp}(\alpha)$, bcc $(\beta)$ and $\omega$ (a 3-atom hexagonal structure) with respect to the fcc phase are displayed in figures 11 and 12 for $\mathrm{Zr}$ and

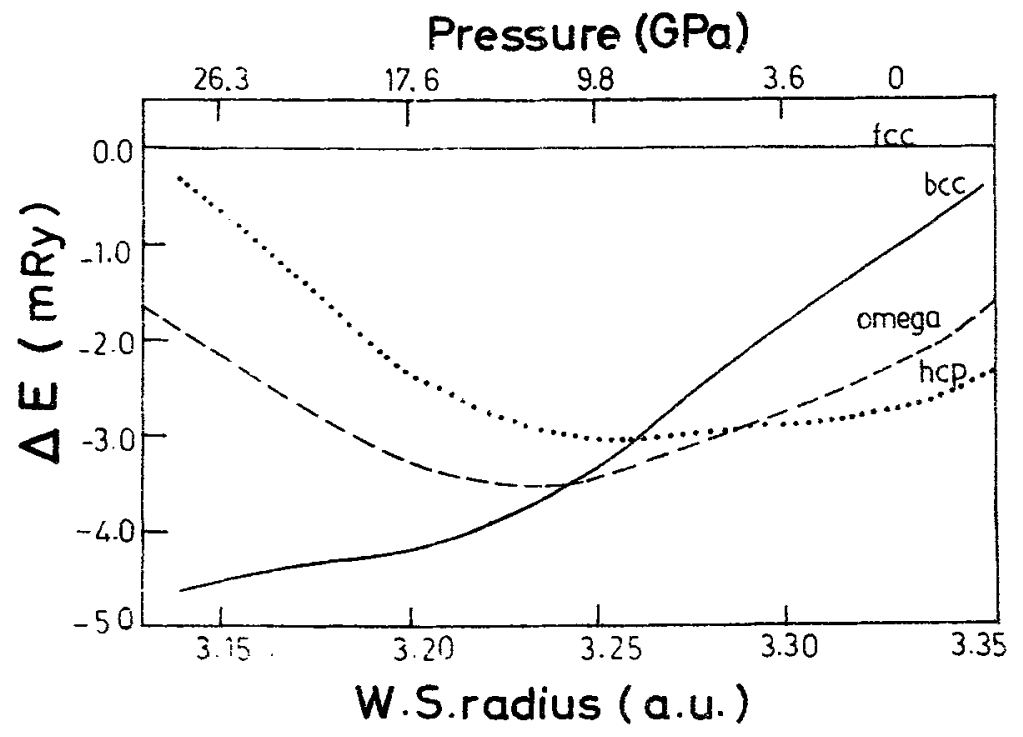

Figure 11. Structural energy differences for $\mathrm{Zr}$. 


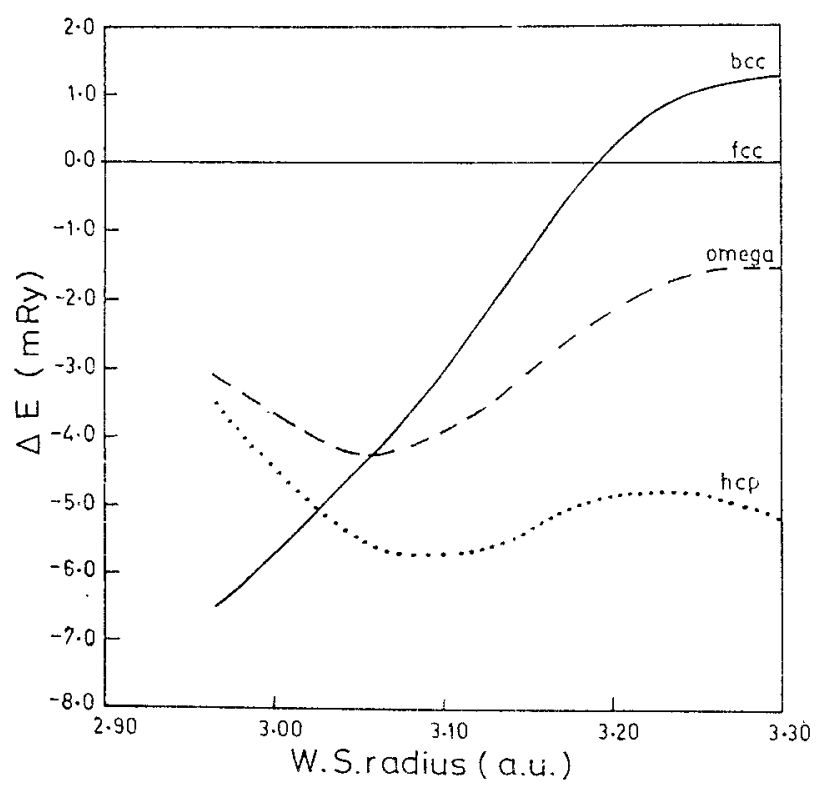

Figure 12. Structural energy differences for Hf.

Hf respectively. The $\beta$ phase was included by noting that the $d$-electron occupancy increases with pressure in these group IV elements and the next element in each of the series is in the bcc phase. These correctly show that the $\alpha$ structure is the lowest energy structure at normal volume and on compression the first transition is from $\alpha$ to the $\omega$ structure. This was already established experimentally for $\mathrm{Zr}$. At higher pressures, we predict new phase transitions (i.e. $\omega-\beta$ ) at 11 and $51 \mathrm{GPa}$ in $\mathrm{Zr}$ and $\mathrm{Hf}$ respectively. Subsequently, these transitions have been detected by the Cornell group using the energy-dispersive X-ray diffraction with a diamond anvil cell (Xia et al $1990 \mathrm{a}, \mathrm{b})$. The experimental transition pressures are $30 \pm 2$ and $71 \pm 1 \mathrm{GPa}$. The agreement between the theoretical and experimental values is within the state of art (Skriver 1985). A similar theoretical analysis on Ti suggested that no $\omega-\beta$ transition occurs in this. This result was confirmed by our own diamond cell experiments and also by the Cornell group.

These one-electron theory triumphs now successfully explain the causes of the experimental shock discontinuities in these metals as due to $\omega-\beta$ in $\mathrm{Zr}$ and $\alpha-\omega$ in $\mathrm{Hf}$ while there is no discontinuity in $\mathrm{Ti}$. The latter has again been confirmed by recent experiments at LLNL (Morris 1991).

\section{References}

Brown J M and McQueen R G 1986 J. Geophys. Res. 917485

Carter W J 1973 in Metallurgical effects of high strain rate (eds) R W Rhode, B M Butcher, J R Holland and C H Karnesi (New York: Plenum)

Carter W J, Fritz J N, Marsh S P and McQueen R G 1975 J. Phys. Chem. Sol. 36741

Chidambaram R, Sikka S K and Gupta S C 1985 Pramana - J. Phys. 24245

Godwal B K, Sikka S K and Chidambaram R 1977 Phys. Rev. B20 2362

Godwal B K, Sikka S K and Chidambaram R 1983 Phys. Rep. 102121 
Guillermet A F 1987 J. Phys. Chem. Sol. 48819

Gupta S C 1980 Some investigations of explosion-induced shock wave phenomena in materials, PhD Thesis, University of Bombay

Gupta S C, Gyanchandani J S, Sikka S K, Chidambaram R, Agarwal R G and Kakodkar A K 1990 Solid State Phys. India C32 445

Gust W H and Royce E B 1973 Phys. Rev. B3 3595

Gyanchandani J S, Gupta S C, Sikka S K and Chidambaram R 1987 in Shock waves in condensed matter (eds) S C Schmidt and N C Holmes (Amsterdam: Elsevier)

Gyanchandani J S, Gupta S C, Sikka S K and Chidambaram R 1989 High Pressure Res. 4472

Gyanchandani J S, Gupta S C, Sikka S K and Chidambaram R 1990a J. Phys. Cond. Matter 2301

Gyanchandani J S. Gupta S C, Sikka S K and Chidambaram R 1990b in Shock compression in condensed matter (eds) S C Schmidt, J N Johnson and L W Davison (Amsterdam: Elsevier)

Gyanchandani J S, Gupta S C, Sikka S K and Chidambaram R 1990c J. Phys. Cond. Matter 26457

Jeanloz R 1987 J. Geophys. Res. 9210352

Johnson Q, Mitchell A, Keeler R N and Evans L 1970 Phys. Rev. Lett. 251099

Kiselov A N and Falkov A A 1982 Fiz. Goreniya $i$ Vzryva 18105

Kutsar A R and German V N 1976 in Third Int. Conf. on Ti, Moscow State Univ., Moscow

Kutsar A R 1982 JETP Lett. 35108

Kutsar A R, Pavlovaskii M N and Komissarov V V 1984 JETP Lett. 39141

Mackintosh A R and Andersen O K 1980 in Electrons, at the Fermi surface (ed.) M Springford (Cambridge: Univ. Press) p. 149

McQueen R G, Marsh S P, Taylor J W, Fritz J N and Carter W J 1970 in High velocity impact phenomena (ed.) R Kinslow (New York: Academic Press)

Minshall S 1955 Phys. Rev. 98271

Morris C E 1991 unpublished

Pashkov P O and Polykova I I 1972 Dokl. Acad. Nauk, SSSR 204332

Pettifor D G 1976 Commun. Phys. 1141

Ross M 1990 High Press. Res. 5683

Sikka S K 1985 Bull. Mater. Sci. 7377

Sikka S K 1986 in Shock waves in condensed matter (ed.) Y M Gupta (New York: Plenum Press)

Sikka S K, Vohra Y K and Chidambaram R 1982 Prog. Mater. Sci. 27245

Skriver H L 1984 in LMTO method (Berlin: Springer)

Skriver H L 1985 Phys. Rev. 311909

Syassen K and Vortmann G 1981 in Physics of solids under pressure (eds) J S Schilling and R N Shelton (Amsterdam: North Holland) p 319--322

Takemura K and Syassen K 1985 J. Phys. F15 543

Wark J S, Whitlock R R, Hauer A, Swain J E and Solore P J 1987 in Shock waves in condensed matter (Amsterdam: Elsevier) p. 781

Xia H, Duclos S J, Ruoff A L and Vohra Y K 1990a Phys. Rev. Lett. 64204

Xia H, Parthasarthy G, Huan L, Vohra Y K and Ruoff A L 1990b Phys. Rev. B42 6736

Zilbershtein V A, Chistotina N P, Zharov A A, Grishina N S and Estrin E I 1975 Fiz. Metal. Metalloved 39445 